\title{
ESTIMATIVA DO CONFORTO DE MATRIZES DE FRANGO DE CORTE BASEADA EM ANÁLISE DO COMPORTAMENTO DE PREFERÊNCIA TÉRMICA
}

\author{
DANILO F. PEREIRA ${ }^{1}$, IRENILZA DE A. NÄÄS ${ }^{2}$
}

RESUMO: Considerando o comportamento social, é sugestivo que a freqüência e a intensidade de interações agressivas, o total de coesão social e compreensão da extensão de vícios sociais possam ser utilizados para avaliação de bem-estar. Este trabalho propõe que a freqüência de utilização de determinados locais dentro do galpão possa ser usada como variável para monitorar estados de bemestar térmico e/ou estresse das aves alojadas. A partir de dados registrados no verão de 2000-2001, esta pesquisa identificou as temperaturas críticas máximas $\left(\mathrm{tc}_{\text {máx }}\right.$ ) de matrizes pesadas, individualmente, por meio de análise estatística da freqüência de uso de locais previamente estabelecidos, utilizando a

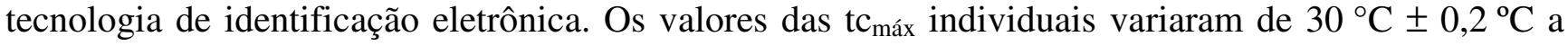
$32,3{ }^{\circ} \mathrm{C} \pm 0,2^{\circ} \mathrm{C}$ e a temperatura crítica máxima média para o grupo foi $30,9^{\circ} \mathrm{C} \pm 0,8^{\circ} \mathrm{C}$.

PALAVRAS-CHAVE: avicultura, zona de termoneutralidade, identificação eletrônica.

\section{IDENTIFICATION OF THE MAXIMUM CRITICAL TEMPERATURE OF FEMALE BROILER BREEDERS BASED ON ANALYSIS OF THEIR THERMAL PREFERENCE BEHAVIOR}

\begin{abstract}
Considering the social behavior, it is suggested that the frequency and the intensity of aggressive interactions, as the well as the total social cohesion, and the understanding of social behavior can be used for welfare evaluation. This research proposes that the frequency of use of specific places inside the bird's housing can be used as variable to monitor thermal welfare status and/or the stress level of the lodged birds. From data registered in the summer of 2000/2001, this study identified the maximum critical temperatures ( $\mathrm{ct}_{\max }$ ) of the female broiler breeders individually, using statistical analysis of the use of previously determined places, applying the technology of electronic identification. The $\mathrm{ct}_{\max }$ values varied from $30{ }^{\circ} \mathrm{C} \pm 0,2{ }^{\circ} \mathrm{C}$ to $32,3{ }^{\circ} \mathrm{C} \pm 0,2{ }^{\circ} \mathrm{C}$ and the critical temperature maximum average for the group was $30,9^{\circ} \mathrm{C} \pm 0,8^{\circ} \mathrm{C}$.
\end{abstract}

KEYWORDS: poultry, thermoneutral zone, electronic identification.

\section{INTRODUÇÃO}

A aquisição de conhecimentos específicos das áreas de nutrição, genética e manejo permitiu que índices positivos fossem alcançados, juntamente com os conhecimentos adquiridos dos fatores de produção relacionados à sanidade e às instalações. Porém, uma atividade com a magnitude da avicultura, que usa equipamentos de última geração e serviços atualizados, é levada a tomar decisões que envolvem todos os aspectos de produção, apoiada em critérios subjetivos. A utilização de equipamentos eletrônicos e sistemas informatizados para coleta, gravação e gerenciamento dos dados de produção são ferramentas que já estão presentes na avicultura, porém existe a necessidade de integrar esses equipamentos a sistemas de suporte a decisão (DSS). A tecnologia já existente de identificação eletrônica (RFID), associada com sensores de respostas fisiológicas que utilizam a

\footnotetext{
${ }^{1}$ Prof. Assistente, UNESP - Câmpus de Tupã - SP, Fone: (0XX14) 3404.4200, Ramal: 4218, Fax: (0XX14) 3404.4201, danilo@tupa.unesp.br

${ }^{2}$ Profa. Titular, FEAGRI/UNICAMP, Campinas - SP, Fone: (OXX19) 3788.1039, Fax: (OXX19) 3788.1010, irenilza@ agr.unicamp.br

Recebido pelo Conselho Editorial em: 2-6-2003

Aprovado pelo Conselho Editorial em: 6-7-2005
}

Eng. Agríc., Jaboticabal, v.25, n.2, p.315-321, maio/ago. 2005 
telemetria como meio de transmissão, interligadas a sistemas inteligentes (redes neurais) de suporte a decisão, constituirá a base dos sistemas de produção animal do futuro.

DUNCAN \& MENCH (1993) propuseram que o comportamento possa ser utilizado para identificar estados de sofrimento do animal e, em particular, os estados de febre, frustração e dor em vários sistemas de produção animal. Pouco conhecida é a maneira pela qual diferentes espécies de aves respondem a outros estados reativos e ao estresse. Considerando o comportamento social, é sugestivo que a freqüência e a intensidade de interações agressivas, o total de coesão social e a extensão de vícios sociais possam ser utilizados para avaliação de bem-estar. Atualmente, os sistemas de climatização de galpão se apóiam simplesmente na identificação da temperatura do ar e, em alguns casos, da umidade relativa. Esses sistemas de climatização não consideram as diferentes capacidades de adaptação ou aclimatação das aves em ambientes estressores. Para isso, o registro de variáveis que indicam o conforto das aves alojadas é fundamental. A necessidade de utilizar respostas biológicas, fisiológicas ou comportamentais das aves alojadas vai além das considerações sobre as capacidades de aclimatação das aves.

Diversos autores estudaram os limites da termoneutralidade de matrizes pesadas e observa-se grande variação entre os limites superiores encontrados. HAHN (1992) relacionou alguns limites superiores da zona de termoneutralidade de matrizes pesadas publicadas por outros autores, sendo que esses valores variaram entre $26,7^{\circ} \mathrm{C}$ e $35^{\circ} \mathrm{C}$. HOCKING et al. (1994), estudando respostas fisiológicas de matrizes pesadas submetidas a altas temperaturas, sugeriram como temperatura crítica valores entre 29 e $30^{\circ} \mathrm{C}$. COSTA (1994) relata estudos baseados em dados de literatura e propõe que, para matrizes pesadas adultas, a temperatura de conforto térmico seja aproximadamente $24^{\circ} \mathrm{C}$, enquanto o limite superior da zona de termoneutralidade seja $29^{\circ} \mathrm{C}$. É importante ressaltar que esses dados se referem às linhagens antigas que estão sendo substituídas por linhagens novas (alta conformação) no mercado atual. Essas variações são atribuídas às diferenças genéticas das aves estudadas em cada trabalho, aos diferentes climas onde foram realizados os estudos e às diferentes capacidades de adaptação (aclimatação) dos animais.

O presente trabalho tem como objetivo estimar a temperatura crítica superior de matrizes pesadas, por meio da análise do comportamento de preferências térmicas, utilizando a tecnologia de identificação eletrônica.

\section{MATERIAL E MÉTODOS}

O experimento foi conduzido no município de Campinas - SP, situado à latitude de $22^{\circ} 53$ ' $S$, altitude de 695 m, tipo climático Cwa, segundo a classificação de Köeppen, sendo caracterizado como clima tropical de altitude, com inverno seco e verão quente, na área experimental do Laboratório de Conforto Ambiental da FEAGRI/UNICAMP, em protótipo de modelo em escala reduzida e distorcida de galpão de produção. Na Tabela 1, são apresentadas as dimensões do modelo em escala reduzida e distorcida utilizadas no experimento.

TABELA 1. Dimensões do modelo em escala natural e distorcida.

\begin{tabular}{llccc}
\hline Escala & & $1: 1$ (natural) & $1: 10$ (horizontal) & $1: 2$ (vertical) \\
\hline Largura & $(\mathrm{m})$ & 14,00 & 1,40 & - \\
Comprimento & $(\mathrm{m})$ & 30,00 & 3,00 & - \\
Pé-direito & $(\mathrm{m})$ & 3,00 & - & 1,50 \\
Altura mureta & $(\mathrm{m})$ & 0,20 & - & 0,10 \\
\hline
\end{tabular}


O modelo em escala reduzida e distorcida, que reproduz as instalações avícolas, foi construído na direção leste-oeste, sendo o seu entorno constituído de área plana coberta de grama. A coleta de dados ocorreu entre o período de $1^{\mathrm{o}}$ de outubro de 2000 e 31 de janeiro de 2001.

Foram utilizadas matrizes de frango de corte da linhagem Hybro- $\mathrm{G}^{\circledR}$. Oito matrizes pesadas, em início de produção, com idade de 28 semanas, foram identificadas eletronicamente através do implante em três locais diferentes, sendo que quatro aves receberam o implante no pé, uma ave recebeu o implante na perna e três aves receberam o implante na coxa (CURTO, 2002), conforme Figura 1a. Segundo PEREIRA et al. (2001), as freqüências absolutas de registros obtidas nos três locais de implante não apresentam diferença estatística para $\alpha=5 \%$.

Os registros para o monitoramento do comportamento das aves foram feitos por meio da tecnologia de identificação eletrônica da Trovan ${ }^{\circledR}$. Essa tecnologia é representada por dois equipamentos: o transponder, que contém um número criptografado e tem o objetivo de identificar o animal que recebe o implante, e a antena (ou leitor), que identifica o número contido no transponder e transmite para um terminal a informação, conforme Figura 1b. Os leitores ou antenas foram responsáveis pelo registro dos dados contidos nos transponders. Estrategicamente colocadas nos protótipos (na passagem debaixo da cama de maravalha entre a parede e o comedouro e debaixo do bebedouro), as antenas permitiram, por meio de conexão com um software, fazer o monitoramento do comportamento das matrizes, por meio da captação da informação contida nos transponders e o relacionamento do evento em que a informação de identificação da ave foi coletada (no bebedouro, no ninho e na passagem) com as condições do ambiente naquele instante.

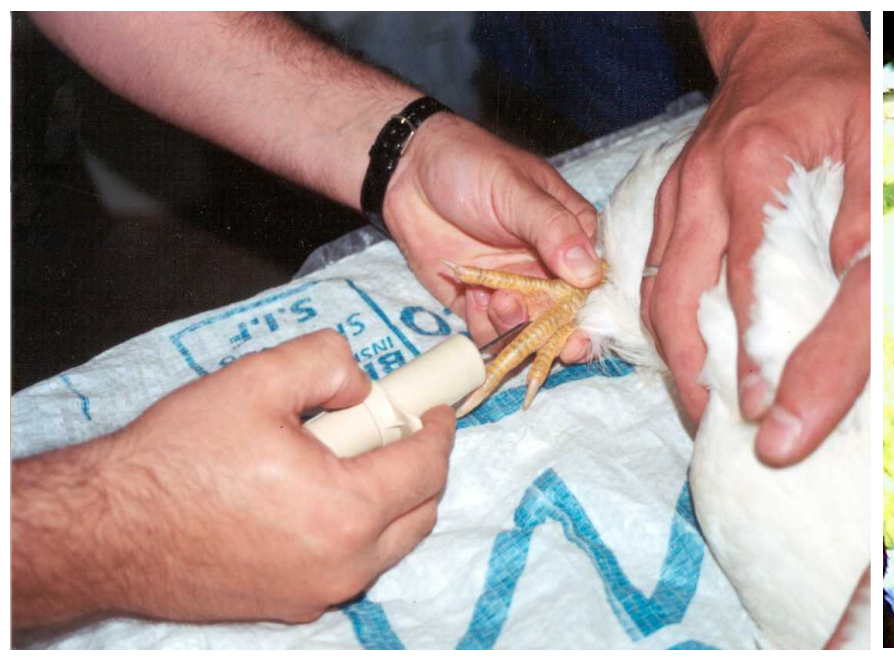

a

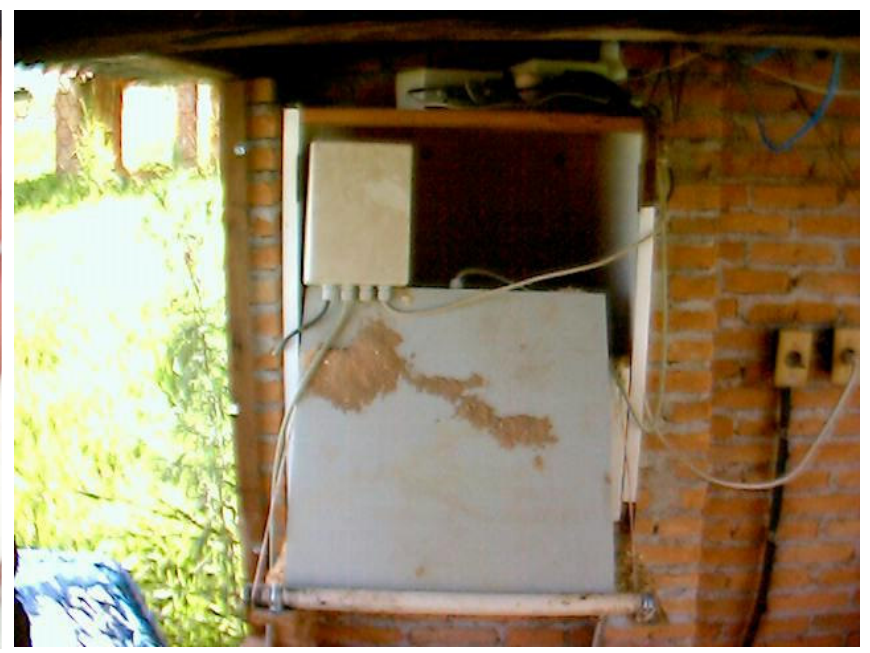

b

FIGURA 1. Foto do implante do transponder na ave (a) e da antena de identificação eletrônica (b).

Foram identificados dois estados de comportamento de uso das aves no interior dos modelos: no bebedouro e atividade motora dentro do galpão (descansando ou não). O experimento foi conduzido de forma a adquirir as informações referentes ao estado em que as aves se encontravam em períodos de 24 horas. Foram feitos registros de informações referentes ao dia e ao horário de coleta, temperatura de bulbo seco (tbs), temperatura de bulbo úmido (tbu) e temperatura de globo negro (tg). Esses dados serviram de referência na avaliação do comportamento em função do ambiente. Para a medida dos dados de ambiente, foram utilizados termopares do tipo J, instalados no centro do galpão onde ocorreu o experimento. Esses termopares transmitiram os dados por meio de cabos para um datalogger da marca Barnant ${ }^{\circledR}$ que converteu o sinal analógico recebido em temperatura, com precisão de $0,05^{\circ} \mathrm{C}$. 
A partir dos dados coletados no experimento, foi gerado um banco de dados utilizando o $M S$ $S Q L$ Server ${ }^{\circledR}$. Foi escolhido esse Banco de Dados pela fácil manipulação dos dados e por permitir a construção de consultas estruturadas (Datamining). Para determinar a temperatura crítica máxima, encontrou-se um padrão comportamental considerado ótimo a partir dos dados de termoneutralidade sugerido por COSTA (1994), para períodos de 24 horas e para cada ave.

Foram construídos, com as temperaturas críticas encontradas para cada ave, um histograma para verificação da distribuição das temperaturas e um intervalo de confiança para a mediana. Considerouse que a temperatura crítica de cada ave variou entre o intervalo de confiança da mediana, para um nível de confiança de $95 \%$. Foram também utilizados teste de comparações múltiplas de médias de Tukey e teste de normalidade de Anderson-Darling.

\section{RESULTADOS E DISCUSSÃO}

As análises foram elaboradas observando-se as diferenças comportamentais entre os indivíduos. As hipóteses levantadas para as análises foram: (1) a temperatura crítica superior do grupo é maior que $29^{\circ} \mathrm{C}$; (2) as aves possuem diferentes temperaturas críticas superiores entre si, devido às diferentes capacidades de adaptação ao ambiente, e (3) é possível identificar, em condições de campo, os limites da termoneutralidade, analisando o comportamento de uso de cada ave em função do ambiente.

O período de coleta apresentou condições ambientais atípicas para o período e a região. É comum, nesses meses de verão, clima quente e úmido, porém, no ano do registros dos dados, ocorreram altas temperaturas e baixas umidades relativas. $\mathrm{O}$ fato de a umidade relativa estar em níveis muito baixos, favoreceu a troca de calor latente pela respiração das aves, contribuindo para melhor troca de calor entre as aves e o ambiente. Devido à capacidade dos homeotermos se adaptarem a climas quentes, este trabalho levanta a hipótese de que o limite superior da termoneutralidade, aqui representada pela temperatura crítica, das matrizes pesadas estudadas, é superior ao proposto pela literatura.

A partir da hipótese de que a $\mathrm{tc}_{\text {máx }}>29^{\circ} \mathrm{C}$, proposta por COSTA (1994), buscaram-se, nos dados coletados entre outubro-2000 e janeiro-2001, os dias em que a temperatura máxima de bulbo seco não ultrapassou os $29^{\circ} \mathrm{C}$, de forma a adquirir o padrão de comportamento de freqüência de uso dos locais pelas aves, dentro da zona de termoneutralidade. Os dias em que a máxima temperatura de bulbo seco (tbs) não ultrapassou os $29^{\circ} \mathrm{C}$ são descritos na Tabela 2.

TABELA 2. Dias em que a tbs máx diária não ultrapassou $29^{\circ} \mathrm{C}$.

\begin{tabular}{ccccccccc}
\hline Dia & $29-10-2000$ & $13-11-2000$ & $15-11-2000$ & $19-11-2000$ & $1^{\circ}-12-2000$ & $22-12-2000$ & $28-12-2000$ & $31-12-2000$ \\
\hline tbs $_{\text {máx }}\left({ }^{\circ} \mathrm{C}\right)$ & 26,9 & 27,1 & 28,2 & 28,2 & 26,8 & 28,8 & 28,2 & 28,6 \\
\hline
\end{tabular}

Determinados os dias em que a tbs não ultrapassou os $29^{\circ} \mathrm{C}$, identificou-se o padrão do comportamento de uso dos locais para cada ave, para esses dias. O comportamento de uso foi avaliado em termos de número absoluto de registros feitos em cada antena, em períodos de 24 horas. Por meio de estatística convencional, foram determinados os intervalos de confiança da mediana, para nível de significância de $\alpha=5 \%$. Os intervalos de confiança são apresentados na Tabela 3.

Os intervalos de confiança mostrados na Tabela 3 indicam o padrão de comportamento de uso de cada local por cada ave, dentro da zona de termoneutralidade. A partir do intervalo de confiança da Tabela 2, buscaram-se, no banco de dados, os dias em que esse padrão se repetiu para cada ave, tanto para a frequiência de uso de bebedouro, como para o uso de passagem, e verificaram-se as máximas tbs ocorridas nesses dias. 
TABELA 3. Intervalos de confiança do comportamento de uso dos locais para os dias em que a tbs $<29^{\circ} \mathrm{C}$.

\begin{tabular}{|c|c|c|c|c|}
\hline \multirow{3}{*}{ Ave } & \multicolumn{4}{|c|}{ Número de Registros } \\
\hline & \multicolumn{2}{|c|}{ Bebedouro } & \multicolumn{2}{|c|}{ Passagem } \\
\hline & Mínimo & Máximo & Mínimo & Máximo \\
\hline 1 & 117 & 608 & 106 & 162 \\
\hline 2 & 96 & 280 & 47 & 185 \\
\hline 3 & 99 & 379 & 87 & 293 \\
\hline 4 & 121 & 337 & 92 & 347 \\
\hline 5 & 113 & 387 & 123 & 375 \\
\hline 6 & 188 & 411 & 47 & 155 \\
\hline 7 & 22 & 334 & 58 & 137 \\
\hline 8 & 125 & 738 & 105 & 160 \\
\hline
\end{tabular}

Foram construídos, para as temperaturas críticas encontradas, um histograma e um teste de normalidade de Anderson-Darling para a verificação da distribuição. Apesar de o teste não rejeitar a hipótese de normalidade, adotaram-se, para as análises, os testes de mediana, devido ao número pequeno de dias que registraram comportamentos de uso dos locais selecionados, com valores de tbs $<29^{\circ} \mathrm{C}$. Portanto, para a identificação das $\mathrm{tc}_{\text {máx }}$ de cada ave, construiu-se um intervalo de confiança de $95 \%$ para a mediana, encontrada para cada ave, conforme Tabela 4. A verificação da temperatura que constitui o limite superior da termoneutralidade dessa ave $\left(\mathrm{tc}_{\text {máx }}\right)$, considerou o padrão ótimo de comportamento de uso para as temperaturas propostas por COSTA (1994) de $24{ }^{\circ} \mathrm{C} \pm 0,5^{\circ} \mathrm{C}<$ tbs $<29^{\circ} \mathrm{C} \pm 0,5^{\circ} \mathrm{C}$. Analisaram-se, ainda, as temperaturas correspondentes ao limite inferior, mediana e o limite superior do intervalo de confiança da mediana, obtidas pela análise estatística.

TABELA 4. Temperaturas utilizadas para análise e identificação da temperatura crítica superior de cada ave, considerando um intervalo de $\pm 0,5^{\circ} \mathrm{C}$.

\begin{tabular}{cccccc}
\hline Ave & $\begin{array}{c}\text { Temperatura de } \\
\text { Conforto } *\end{array}$ & $\begin{array}{c}\text { Temperatura Crítica } \\
\text { Máxima }\end{array}$ & $\begin{array}{c}\text { Limite Inferior do } \\
\text { IC da Mediana }\end{array}$ & Mediana & $\begin{array}{c}\text { Limite Superior do } \\
\text { IC da Mediana }\end{array}$ \\
\hline 1 & 24,0 & 29,0 & $29,2{ }^{\circ} \mathrm{C}$ & $30,6^{\circ} \mathrm{C}$ & $32,2^{\circ} \mathrm{C}$ \\
2 & 24,0 & 29,0 & $30,7{ }^{\circ} \mathrm{C}$ & $31,7{ }^{\circ} \mathrm{C}$ & $32,1^{\circ} \mathrm{C}$ \\
3 & 24,0 & 29,0 & $30,5{ }^{\circ} \mathrm{C}$ & $31,2{ }^{\circ} \mathrm{C}$ & $32,0^{\circ} \mathrm{C}$ \\
4 & 24,0 & 29,0 & $31,2{ }^{\circ} \mathrm{C}$ & $32,0{ }^{\circ} \mathrm{C}$ & $32,5^{\circ} \mathrm{C}$ \\
5 & 24,0 & 29,0 & $30,4{ }^{\circ} \mathrm{C}$ & $31,9^{\circ} \mathrm{C}$ & $32,3^{\circ} \mathrm{C}$ \\
6 & 24,0 & 29,0 & $30,3{ }^{\circ} \mathrm{C}$ & $31,7{ }^{\circ} \mathrm{C}$ & $32,3^{\circ} \mathrm{C}$ \\
7 & 24,0 & 29,0 & $29,1{ }^{\circ} \mathrm{C}$ & $30,1^{\circ} \mathrm{C}$ & $31,7^{\circ} \mathrm{C}$ \\
8 & 24,0 & 29,0 & $29,1^{\circ} \mathrm{C}$ & $30,4^{\circ} \mathrm{C}$ & $32,3^{\circ} \mathrm{C}$ \\
\hline
\end{tabular}

* Proposta por COSTA (1994)

Para a análise estatística das temperaturas acima do limite superior de termoneutralidade, buscou-se o comportamento de uso dos mesmos locais pelas aves, para essas temperaturas, em períodos de 15 dias. A partir dos resultados de freqüência de utilização obtidos para cada temperatura desse período, verificou-se a existência de diferença nos comportamentos de uso dos locais entre as temperaturas para cada ave. Utilizou-se do teste de comparações múltiplas de Tukey, para nível de significância de $\alpha=5 \%$, identificando qual a temperatura que teve influência significativa no comportamento das aves, individualmente. Identificada a temperatura que influi nesse comportamento de uso, adotou-se a temperatura imediatamente inferior, dentro da análise, como a temperatura crítica 
superior dessa ave. Na Tabela 5, apresentam-se as temperaturas críticas superiores, constituindo o limite superior da zona de termoneutralidade, encontradas para cada ave, e a média do grupo.

TABELA 5. Limites superiores de temperatura da zona de termoneutralidade (LS-ZTN) encontrados para as aves.

\begin{tabular}{cc}
\hline Ave & LS-ZTN \\
\hline 1 & 30,6 \\
2 & 31,7 \\
3 & 30,5 \\
4 & 31,2 \\
5 & 30,4 \\
6 & 32,3 \\
7 & 30,1 \\
8 & 30,4 \\
\hline Média \pm Desvio-Padrão & $30,9 \pm 0,8$ \\
\hline
\end{tabular}

Observa-se, na Tabela 5, que as máximas temperaturas encontradas são diferentes entre as aves. Esses resultados confirmam a hipótese inicial de que as aves apresentam diferenças quanto aos limites da termoneutralidade, sendo indício de que existe diferença entre as aves quanto à resistência térmica ao ambiente.

O intervalo de confiança de $95 \%$ construído para a média obtida pelas temperaturas críticas máximas de cada ave foi $\left(30,5^{\circ} \mathrm{C} ; 31,3{ }^{\circ} \mathrm{C}\right)$, tendo sido superior aos valores propostos por COSTA (1994) e HOCKING et al. (1994), confirmando as hipóteses iniciais: (1) a temperatura crítica superior do grupo é superior a $29^{\circ} \mathrm{C}$ e (2) as aves possuem diferentes temperaturas críticas superiores entre si, variando entre $30,4^{\circ} \mathrm{C}$ e $31,7^{\circ} \mathrm{C}$, com desvio-padrão de $0,8^{\circ} \mathrm{C}$.

\section{CONCLUSÕES}

Foi possível identificar diferenças comportamentais de matrizes pesadas quanto à freqüência de utilização de bebedouro e passagem em relação às condições do ambiente, por meio do sistema de monitoramento proposto.

Estimaram-se, a partir da metodologia de análise proposta por este trabalho, as temperaturas críticas máximas de cada indivíduo, sendo que o valor individual variou entre 30,0 $\pm 0,2$ e 32,2 $\pm 0,2$ ${ }^{\circ} \mathrm{C}$ e a temperatura crítica máxima média para o grupo foi $30,9 \pm 0,8{ }^{\circ} \mathrm{C}$.

A viabilidade da utilização da tecnologia de identificação eletrônica para registro de dados de freqüência de uso de determinados locais, por matrizes pesadas, pode constituir ferramenta importante para melhor compreensão do conforto térmico, a partir da sua preferência térmica.

A partir do registro de dados de comportamento de uso de locais determinados, criou-se uma base de informações que sugerem novos limites para o bem-estar das aves monitoradas, servindo de base de conhecimento para acionamento de sistemas de climatização em alojamento de matrizes Hybro- $\mathrm{G}^{\circledR}$. As temperaturas mais altas do que as recomendadas pela literatura, para manter a zona de conforto na produção, permitem redução do uso de energia elétrica, sem que se deixe de atender às demandas internacionais de bem-estar animal. 


\section{REFERÊNCIAS}

COSTA, C.A. Pontos críticos do manejo de matrizes. In: CONFERÊNCIA APINCO DE CIÊNCIA E TECNOLOGIA AVÍCOLA: MANEJO DE MATRIZES, 1994, Campinas. Anais... Campinas: Fundação Apinco de Ciência e Tecnologia Avícolas, 1994. p.1-10.

CURTO, F.P.F. Estudo do comportamento de matrizes pesadas (frango de corte), em diferentes ambientes utilizando identificação eletrônica e rádio-freqüência. 2002. 135 f. Tese (Doutorado em Construções Rurais e Ambiência) - Faculdade de Engenharia Agrícola, Universidade Estadual de Campinas, Campinas, 2002.

DUNCAN, I.J.H.; MENCH, J.A. Behaviour as an indicator of welfare in various systems. In: EUROPEAN SYMPOSIUM ON POULTRY WELFARE, 4., 1993, Potters Bar. Proceedings... Potters Bar: Universities Federation for Animal Welfare, 1993. p.69-80.

HAHN, G.L. Housing for cattle, sheep and poultry in the tropics. In: YOUSEF, M.R. (Ed.) Animal production in the tropics. New York: Praeger, 1982. p.43-72.

HOCKING, P.M.; MAXWELL, M.H.; MITCHEL, M.A. Haematology and blood composition at two ambient temperatures in genetically fat and lean adult broiler breeder females fed ad libitum or restricted throughout life. British Poultry Science, Edinburgh, v.35, p.799-807, 1994.

PEREIRA, D.F.; NÄÄS, I.A.; CURTO, F.P.F.; MURAYAMA, M. Avaliação do local do implante de microchip utilizados para identificação eletrônica em matrizes pesadas (Evaluating the place for transponder implant used in EID for broiler's breeder). Brazilian Journal of Poultry Science, Campinas, v.1, n.3, p.53, 2001. Suplement 